\title{
Inflations of anti-cycles and Hadwiger's Conjecture
}

\author{
Anders Sune Pedersen, Michael D. Plummer, and Buarne Toft \\ Dedicated to Adrian Bondy on his 70th birthday
}

\begin{abstract}
Let $G$ be any graph. An inflation of a graph $G$ is obtained from $G$ first by replacing each of its vertices with either the empty set or a complete graph, and then whenever two vertices $x$ and $y$ are adjacent in $G$ we join each vertex of the replacement clique for $x$ to each vertex of the replacement clique for $y$ by an edge.

Let $G$ be any inflation of the complement of an odd cycle. It is shown that $(1) \chi(G)=\max \{\omega(G),\lceil|V(G)| / 2\rceil\}$ and $(2) G \succeq K_{\chi(G)}$ by a short direct proof; that is to say, Hadwiger's Conjecture holds for $G$. We present a comprehensive survey of the area surrounding these results.
\end{abstract}

Keywords AND PHRASES: Hadwiger's Conjecture, inflation, coloring, chromatic number, perfect graph, anti-cycle, quasi-line graph, vertexcritical, edge-critical.

\section{Introduction}

Hadwiger's Conjecture [9] from 1943 suggests a far reaching generalization of the Four Color Theorem, namely that any $k$-chromatic graph has the complete graph $K_{k}$ as a minor.

Wolfgang Mader and Paul Seymour were the first persons to stress that an important special case of Hadwiger's Conjecture is obtained by adding the assumption that the independence number of the graph is no greater than 2. At first glance, this class of graphs may seem too restricted to be of interest until one realizes that the complements of such graphs are the triangle-free graphs, a graph class which has received considerable attention over the years.

By a result of Kim [11] it is known that there is a constant $c$ such that there exist graphs $G$ on $n$ vertices with $\alpha(G) \leq 2$, and having clique number $\omega(G) \leq c \sqrt{n \log n}$. But since $\chi(G) \geq n / 2$ for these graphs, Hadwiger's Conjecture would imply that we can contract $G$ to $K_{\lceil n / 2\rceil}$, a clique much larger in size than a largest clique in $G$.

The special case of Hadwiger's Conjecture in which $\alpha(G) \leq 2$ seems to have been first considered in print by Duchet and Meyniel [6]. 
Chudnovsky and Seymour [5] proved that Hadwiger's Conjecture is true when $\alpha(G) \leq 2$ and $G$ contains a clique on at least $|V(G)| / 4$ vertices if $|V(G)|$ is even, and at least $(\mid(V(G) \mid+3) / 4$ vertices if $|V(G)|$ is odd.

In the present paper, we shall be concerned with inflations of a graph $G$. Such a graph is obtained from $G$ by replacing each of its vertices with either the empty set or a complete graph, and then whenever two vertices $x$ and $y$ are adjacent in $G$ we join each vertex of the replacement clique for $x$ to each vertex of the replacement clique for $y$ by an edge. The replacement cliques will be called "blobs". If $\alpha=2$ in the original graph, then any inflation of the graph also satisfies $\alpha=2$.

The $k$-chromatic graphs which have $K_{k}$ as a subgraph and which, for all $t<k$, have $K_{t}$-subgraphs in all their induced $t$-chromatic subgraphs are called perfect. Lovász [15] proved that inflations of perfect graphs are perfect. It is not known if a similar inflation result holds for graphs satisfying Hadwiger's Conjecture. However, if $G$ is a path, an even cycle or the complement of a path or even cycle, then $G$ is perfect. Hence any inflation $H$ of such a graph is also perfect and satisfies $\chi(H)=\omega(H)$ and Hadwiger's Conjecture is thus trivially satisfied.

In [18] it was proved that Hadwiger's Conjecture holds for all inflations of graphs $G$ having independence number $\alpha(G) \leq 2$ and $|V(G)| \leq 11$. In [17] it was proved that Hadwiger's Conjecture holds for all inflations of the Petersen graph, and in [2] more generally that it holds for all inflations of 3-chromatic graphs.

A graph $G$ is a quasi-line graph if the neighborhood of every vertex can be partitioned into two cliques. (Note that there may be edges joining the two cliques.) These graphs are characterized in [4] and constitute a superclass of the class of line graphs. Chudnovsky and Ovetsky Fradkin [3] proved that Hadwiger's Conjecture holds for all quasi-line graphs thus generalizing an earlier result of Reed and Seymour [19] for line graphs of multi-graphs. The class of quasi-line graphs is closed under making inflations, and hence Hadwiger's Conjecture holds for any inflation of a quasi-line graph. Since every cycle and complement of a cycle (and inflation of such a graph) is a quasi-line graph, it follows that any inflation of a cycle or a complement of a cycle also satisfies Hadwiger's Conjecture.

If $G$ is any inflation of an odd cycle $C_{n}$, then $\chi(G)=\max \{\omega(G)$, $\lceil(2|V(G)| /(n-1)\rceil\}$. That $\chi(G)$ is at least this maximum follows from the fact that $\alpha(G) \leq(n-1) / 2$. The equality was first obtained by T. Gallai (oral communication, 1969). This result appeared in print as Exercise $5 i$ (page 35) in [7], as Theorem 14.1.4 in [16] and it also follows from Theorem 1 in [14]. This theorem, due to T. Gallai, characterizes when an inflation 
$G$ of an odd cycle $C_{n}$ is an edge-critical graph and in fact the characterization for vertex-criticality is the same. In the case when all blobs are nonempty, if we write $n=2 k+1$, this characterization amounts to saying that any two neighboring blobs together have size at most $k-1$ and $|V(G)|=$ $(k-1)((n-1) / 2)+1$.

In this paper we consider inflations of the other type of minimal nonperfect graphs, namely the complements $\overline{C_{2 p+1}}$ of the odd cycles (also known as anti-cycles). We obtain a formula for their chromatic number and give a direct proof that they satisfy Hadwiger's Conjecture. Moreover, we characterize when such a graph is vertex-critical.

We shall adopt the following terminology and notation. A clique in a graph $G$ is a maximal set of pairwise adjacent vertices in $G$. The size of a largest clique in $G$ is denoted by $\omega(G)$. The chromatic number of $G$ is denoted by $\chi(G)$. We write $G \succeq H$ if $G$ contracts to $H$, that is if $H$ can be obtained from $G$ by deleting edges and vertices and contracting edges. A graph $G$ is vertex-(chromatic)-critical (or simply vertex-critical) if $\chi(G-x)<\chi(G)$ for every vertex $x \in V(G)$. A graph $G$ is perfect if $\chi(H)=\omega(H)$, for every induced subgraph $H$ of $G$.

\section{Main results}

In this section we begin by deriving a formula for the chromatic number of an inflation of the complement of an odd cycle of length at least 5 . Such a graph $G$ clearly has $\alpha(G) \leq 2$.

Let $G$ be any graph with $\alpha(G) \leq 2$ and $\chi(G)=k$. Then for such a graph, we note two obvious facts: (i) $k \geq \omega(G)$ and (ii) $k \geq\lceil|V(G)| / 2\rceil$.

Theorem 2.1. Suppose $p \geq 2$ and suppose $G$ is an inflation of $\overline{C_{2 p+1}}$. Then $\chi(G)=\max \{\omega(G),\lceil|V(G)| / 2\rceil\}$.

Proof: Let $H$ be $k$-vertex-critical subgraph of $G$, where $k=\chi(G)$. Then $H$ is also an inflation of $\overline{C_{2 p+1}}$.

Case 1: Suppose one of the blobs in $H$ is empty. Then $H$ is an inflation of the complement of a path. But a path, being bipartite, is perfect. Hence by [15] the complement of this path is also perfect. But also by [15] the inflation of a perfect graph is itself perfect. That is, $H$ is perfect. Hence $\chi(H)=\omega(H)$ and hence $k=\omega(H) \leq \omega(G)$ and hence by (i), $k=\omega(G)$ and, since $p \geq 2$, by (ii) we also have $k=\max \{\omega(G),\lceil|V(G)| / 2\rceil\}$ and the result is true in Case 1.

Case 2: Suppose, then, that no blob in $H$ is empty. 
Let $x$ be any vertex of $H$. Then $H-x$ has at most $2 \chi(H-x)=2(k-1)=$ $2 k-2$ vertices, since at most two vertices can be colored the same color.

Case 2.1: Suppose first that $|V(H)|=2 k-1$. Then $k=\lceil|V(H)| / 2\rceil \leq$ $\lceil|V(G)| / 2\rceil$. But we know that $k \geq \omega(G)$ and by (ii), $k=\lceil|V(G)| / 2\rceil$ and it follows that $\chi(G)=\max \{\omega(G),\lceil|V(G)| / 2\rceil\}$ as desired.

Case 2.2: So suppose $|V(H)| \leq 2 k-2$. Then $\bar{H}$ is disconnected by Gallai [8] (Satz $E_{2} .1$ ). and hence $H$ consists of the join of two non-empty critical subgraphs $H_{1}$ and $H_{2}$. Let the blobs of $H$ be designated $B_{1}, B_{2}, \ldots, B_{2 p+1}$ in clockwise cyclic order.

Clearly each blob $B_{i}$ lies either entirely within $H_{1}$ or $H_{2}$. Suppose, without loss of generality, that $B_{1} \subseteq H_{1}$. Then it is easy to see that $B_{2} \subseteq H_{1}$ and, continuing, all the $B_{i}$ must lie in $H_{1}$; i.e., $H_{2}=\emptyset$, a contradiction. This completes the proof of Theorem 2.1.

Remark 2.2. The above result clearly fails to hold when $p=1$, for just consider the trivial inflation of $\overline{K_{3}}$ in which each blob has size 1.

Remark 2.3. The fractional chromatic number $\chi^{*}(G)$ of a graph $G$ is defined by $\chi^{*}(G)=\min _{q}\left(\chi\left(G\left[K_{q}\right]\right) / q\right.$, where $G\left[K_{q}\right]$ represents the inflation of $G$ obtained by replacing each vertex by a copy of $K_{q}$. A perfect graph $G$ has $\chi^{*}(G)=\chi(G)$; for example, $\chi^{*}\left(C_{2 p}\right)=2$ and $\chi^{*}\left(\overline{C_{2 p}}\right)=p$. For $2 p+1 \geq 5$, Gallai's result mentioned earlier implies that $\chi^{*}\left(C_{2 p+1}\right)=2+1 / p$ and 2.1 implies that $\chi^{*}\left(\overline{C_{2 p+1}}\right)=p+1 / 2$.

Although the next theorem follows from the characterization of quasiline graphs found in [3] and also from the result in [5] mentioned above, we present a direct proof, as it is brief.

Theorem 2.4. If $p \geq 1$ and $G$ is an inflation of $\overline{C_{2 p+1}}$, then $G \succeq K_{\chi(G)}$.

Proof: If $p=1$, the result is clearly true, so henceforth we suppose that $p \geq 2$.

The proof now proceeds by induction on $|V(G)|$. Suppose $|V(G)| \leq 2 p$. Then there must be an empty blob and, as argued in the proof of Theorem 2.1, $G$ must be perfect. But then $\chi(G)=\omega(G)$ and hence $G$ contains $K_{\omega(G)}$, so $G \succeq K_{\omega(G)}=K_{\chi(G)}$.

Now suppose that $|V(G)| \geq 2 p+1$ and that the theorem is true for all graphs with fewer than $|V(G)|$ vertices. Let $K_{\omega}$ be a maximum clique in $G$. Relabelling the blobs if necessary, without loss of generality we may assume that $V\left(B_{1}\right) \subseteq V\left(K_{\omega}\right)$. Hence $V\left(B_{2}\right) \cap V\left(K_{\omega}\right)=\emptyset$. Choose $x \in V\left(B_{2}\right)$. Let $B_{p+2}$ and $B_{p+3}$ be the "opposite" pair of blobs from $B_{2}$. At least one of these two blobs is not in $K_{\omega}$. Choose $y$ in such a blob. Then $K_{\omega} \subseteq G-x-y$. 
If any of $B_{1}, B_{2}, \ldots, B_{2 p+1}$ is empty, then arguing as before, $G$ is perfect and therefore $\chi(G)=\omega(G)$. But then $G$ contracts to $K_{\chi(G)}$ as desired. So suppose that all $2 p+1$ blobs are non-empty.

Then edge $x y \in E(G)$. Moreover, the graph $G-x-y$ is also an inflation of $\overline{C_{2 p+1}}$ and $\omega(G-x-y)=\omega(G)$.

Let us first suppose that $p \geq 3$. Applying the induction hypothesis to $G-x-y$, we have that $G-x-y \succeq K_{\chi(G-x-y)}$. But then $G \succeq K_{\chi(G-x-y)+1}$ where the extra vertex results from the contraction of the edge $x y$. (Note that here we use the assumption that $p \geq 3$.)

It remains to show that $\chi(G-x-y)+1 \geq \chi(G)$. By 2.1 , this inequality may be rewritten as

$$
\max \{\omega(G-x-y),\lceil|V(G)| / 2\rceil-1\}+1 \geq \max \{\omega(G),\lceil|V(G)| / 2\rceil\},
$$

or, since $\omega(G-x-y)=\omega(G)$,

$$
\max \{\omega(G),\lceil|V(G)| / 2\rceil-1\}+1 \geq \max \{\omega(G),\lceil|V(G)| / 2\rceil\},
$$

that is,

$$
\max \{\omega(G)+1,\lceil|V(G)| / 2\rceil\} \geq \max \{\omega(G),\lceil|V(G)| / 2\rceil\} .
$$

But this is obvious.

Finally, suppose that $p=2$. That is to say, $G$ is an inflation of $\overline{C_{5}}$. But $\overline{C_{5}} \cong C_{5}$. So it remains to show that any inflation $G$ of $C_{5}$ contracts to $K_{\chi(G)}$. However, in Pedersen's paper [17] there is a complete proof that (a) if $G$ is any inflation of the 5-cycle, then $\chi(G)=\max \{\omega(G),\lceil|V(G)| / 2\rceil\}$ and (b) $G \succeq K_{\chi(G)}$; that is, Hadwiger's Conjecture holds for $G$. (Lemma 3 and Corollary 5 , respectively.)

(Alternatively, the result in this $p=2$ case follows from much stronger results in [18].)

Remark 2.5. The Hadwiger number of an inflation of $\overline{C_{2 p+1}}$, that is, the largest $h$ for which the graph has $K_{h}$ as a minor, is not always equal to the chromatic number of the graph. The graph shown in Figure 1 is 5-chromatic, but if the edges ek,bh,bd and dh are contracted, a $K_{6}$-minor is obtained.

One can ask also when the inflation of the complement of an odd cycle is $k$-vertex-critical. The next result provides an answer to this question.

Theorem 2.6. Suppose $p \geq 2$ and let $G$ be an inflation of $\overline{C_{2 p+1}}$. Then $G$ is $k$-vertex-critical and different from $K_{k}$ if and only if 


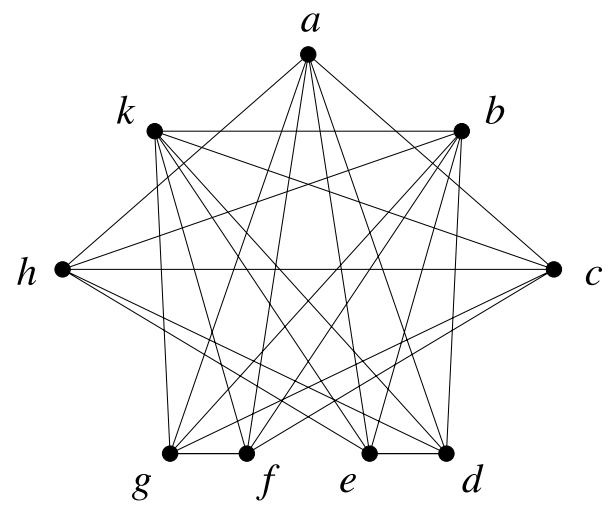

Figure 1: An inflation of $\overline{C_{7}}$.

(a) $k>\omega(G)$ and

(b) $|V(G)|=2 k-1$.

Proof: Suppose that $G$ is $k$-vertex-critical and $G \neq K_{k}$.

Part (a) is clear, so let us consider part (b).

From Theorem 2.1 and part (a), we have that $k=\max \{\omega(G)$, $\lceil|V(G)| / 2\rceil\}=\lceil|V(G)| / 2\rceil$, and hence $|V(G)|=2 k-1$ or $|V(G)|=2 k$. Now choose any $x \in V(G)$. Since $\chi(G-x)=k-1$ and $\alpha(G-x) \leq 2$, we have $|V(G-x)| \leq 2 k-2$. Hence $|V(G)| \leq 2 k-1$. This proves part (b).

Let us now consider the converse. $G \neq K_{k}$ by part (b). Since $k>\omega(G)$, we have by Theorem 2.1 that $k=\chi(G)=\max \{\omega(G),\lceil|V(G)| / 2\rceil\}=$ $\lceil|V(G)| / 2\rceil$. Now choose $x \in V(G)$. Then, again using Theorem 2.1 and the fact that $G-x$ is also the inflation of an anti-cycle, $\chi(G-x)=$ $\max \{\omega(G-x),\lceil|V(G-x)| / 2\rceil\} \leq \max \{\omega(G), k-1\}<k$. That is, $G$ is $k$-vertex-critical.

Remark 2.7. One might further ask if the vertex-critical inflations of $\overline{C_{2 p+1}}$ are also edge-critical. This is true for inflations of $C_{2 p+1}$. However, this is not true for inflations of $\overline{C_{2 p+1}}$ and the graph in Figure 1 is a counterexample. This graph is 5-vertex-critical, but not 5-edge-critical. However, if the four edges af,ag, bh and ck are deleted, a 5-edge-critical graph is obtained. In fact, the graph so obtained is a 9-vertex inflation of $\overline{C_{5}}$ in which four of the five vertices of $\overline{C_{5}}$ are replaced by complete 2-graphs.

Remark 2.8. The dodecahedron is a 3-chromatic graph and hence by the theorem of Casselgren and Pedersen [2], Hadwiger's Conjecture holds for all inflations of the dodecahedron. Is the chromatic number of an inflation of 
the dodecahedron always equal to the chromatic number of one of the inflated 5-cycles? Equivalently, if an inflation of the dodecahedron is k-chromatic, is there a $k$-critical subgraph equal to one of the inflated 5-cycles? This question seems to be unsolved.

More generally, one could try to characterize those $k$-chromatic inflations of 3 -chromatic graphs which have a $k$-critical inflated odd cycle as a subgraph. There are 3 -chromatic graphs with $k$-chromatic inflations not having this property. An example may be found in [2].

\section{Appendix}

One referee suggested a different approach for proving Theorems 2.1 and 2.6 based on matching theory. This alternative proof is based upon the following lemma.

Lemma: Let $G$ be an inflation $\overline{C_{2 p+1}}$, for some $p \geq 2$. if $\omega \leq|V(G)| / 2$, then $\bar{G}$ contains either a hamiltonian cycle or a 2 -factor with only even cycles.

The proof, based on Hall's Theorem, is elementary, but not trivial. Our Theorems 2.1 and 2.6 can then be derived as corollaries of this lemma.

\section{References}

[1] J. Blasiak, A special case of Hadwiger's conjecture, J. Combin. Theory Ser. B 97 (2007) 1056-1073. MR2354718

[2] C. J. Casselgren and A. S. Pedersen, Hadwiger's conjecture for inflations of 3-chromatic graphs, European J. Combin. 51 (2016) 99-108. MR3398842

[3] M. Chudnovsky and A. O. Fradkin, Hadwiger's conjecture for quasi-line graphs, J. Graph Theory 59 (2008) 17-33. MR2432884

[4] M. Chudnovsky and P. Seymour, Claw-free graphs. VII. Quasi-line graphs, J. Combin. Theory Ser. B 102 (2012) 1267-1294. MR2992979

[5] M. Chudnovsky and P. Seymour, Packing seagulls, Combinatorica 32 (2012) 251-282. MR2965277

[6] P. Duchet and H. Meyniel, On Hadwiger's number and the stability number, Ann. Discrete Math. 13 (1982) 71-74. MR0671905

[7] S. Fiorini and R. J. Wilson, Edge-colourings of graphs, Research Notes in Mathematics, No. 16, Pitman, London, 1977. MR0543798 
[8] T. Gallai, Kritische Graphen II, Publ. Math. Inst. Hung. Acad. 8 (1963) 373-395. MR0188100

[9] H. Hadwiger, Über eine Klassifikation der Streckenkomplexe, Vierteljahrsschrift der Naturf. Gesellschaft in Zürich 88 (1943) 133-142. MR0012237

[10] K. Kawarabayashi, M. D. Plummer and B. Toft, Improvements of the theorem of Duchet and Meyniel on Hadwiger's conjecture, J. Combin. Theory Ser. B 95 (2005) 152-167. MR2156345

[11] J. H. Kim, The Ramsey number $R(3, t)$ has order of magnitude $t^{2} / \log t$, Random Struct. Algorithms 7 (1995) 173-207. MR1369063

[12] M. Kriesell, Large connected dominating matchings in $\overline{K_{3}}$-free $\overline{K_{2,3}}$-free graphs, preprint, 2009.

[13] M. Kriesell, On Seymour's strengthening of Hadwiger's conjecture for graphs with certain forbidden subgraphs, Discrete Math. 310 (2010) 2714-2724. MR2672219

[14] U. Krusenstjerna-Hafstrøm and B. Toft, Some remarks on Hadwiger's conjecture and its relation to a conjecture of Lovász, The theory and applications of graphs (Kalamazoo, Michigan, 1980), Wiley, New York, 1981, 449-459. MR0634546

[15] L. Lovász, Normal hypergraphs and the perfect graph conjecture, Discrete Math. 2 (1972) 253-267. MR0302480

[16] O. Ore, The four-color problem, Pure and Applied Mathematics, Vol. 27. Academic Press, New York, 1967, 241. MR0216979

[17] A. S. Pedersen, Hadwiger's conjecture and inflations of the Petersen graph, Discrete Math. 312 (2012) 3537-3543. MR2979482

[18] M. D. Plummer, M. Stiebitz and B. Toft, On a special case of Hadwiger's conjecture, Discussiones Math. 23 (2003) 333-363. MR2070161

[19] B. Reed and P. Seymour, Hadwiger's conjecture for line graphs, European J. Combin. 25 (2004) 873-876. MR2079904

Anders Sune Pedersen

Department of Mathematics \& Computer Science

University of Southern Denmark

ODENSE

DENMARK

E-mail address: asp@imada.sdu.dk 
Michael D. Plummer

Department of Mathematics

VANDERBILT UNIVERSITY

NASHVILLE, TN

USA

E-mail address: Michael.D.Plummer@Vanderbilt.edu

BJARNE TOFT

Department of Mathematics \& Computer Science

University of Southern Denmark

ODENSE

DENMARK

E-mail address: btoft@imada.sdu.dk

Received 20 OCtOBER 2014 